\title{
Comparison of 2-Dimensional and 3-Dimensional Conformal Treatment Plans in Gastric Cancer Radiotherapy
}

\author{
Yasemin Guzle Adas ${ }^{1 *}$, Meltem Nalca Andrieu², Ayse Hicsonmez ${ }^{2}$,Tugba Atakul², \\ Bahar Dirican ${ }^{3}$, Caner Aktas², Sercan Yilmaz ${ }^{4}$, Serap Akyurek², Saban Cakir \\ Gokce $^{2}$, Salih Ergocen ${ }^{5}$
}

\begin{abstract}
Background: Postoperative chemoradiotherapy is accepted as standard treatment for stage IB-IV, M0 gastric cancer. Radiotherapy (RT) planning of gastric cancer is important because of the low radiation tolerance of surrounding critical organs. The purpose of this study was to compare the dosimetric aspects of 2-dimensional (2D) and 3-dimensional (3D) treatment plans, with the twin aims of evaluating the adequacy of 2D planning fields on coverage of planning target volume (PTV) and 3D conformal plans for both covering PTV and reducing the normal tissue doses. Materials and Methods: Thirty-six patients with stage II-IV gastric adenocarcinoma were treated with adjuvant chemoradiotherapy using 3DRT. For each patient, a second 2D treatment plan was generated. The two techniques were compared for target volume coverage and dose to normal tissues using dose volume histogram (DVH) analysis. Results: 3DRT provides more adequate coverage of the target volume. Comparative DVHs for the left kidney and spinal cord demonstrate lower radiation doses with the 3D technique. Conclusions: 3DRT produced better dose distributions and reduced radiation doses to left kidney and spinal cord compared to the 2D technique. For this reason it can be predicted that 3DRT will result in better tumor control and less normal tissue complications.
\end{abstract}

Keywords: Gastric cancer - radiotherapy - computer assisted radiotherapy planning - chemoradiotherapy

Asian Pac J Cancer Prev, 15 (17), 7401-7405

\section{Introduction}

Gastric cancer is the fourth common cancer worldwide and is the second leading cause of death from cancer (Qurieshi et al., 2011; Zare et al., 2013; Calik et al., 2014; Oh et al., 2014). At present complete resection is the only curative therapy (Yu et al., 2013). 25-40\% of the patients are suitable for curative surgery. Postoperative local recurrence is an important issue; $38 \%$ loco regional recurrence and $28 \%$ peritoneal spread are observed post operatively (Czito et al., 2013). Intergroup 0116 study (INT 0116) demonstrated significance of post-operative RT in gastric cancer. Following a 10 year-follow-up period, a significant benefit was observed in relapse free survival and in overall survival (Smalley et al., 2012). Based on the results of this study, post-operative chemoradiotherapy became a standard procedure in stage IB-IV M0 patients. Because of the critical organs in the vicinity, which are low in tolerance, planning of RT in gastric cancer is important (Czito et al., 2013). In this study $2 \mathrm{D}$ and $3 \mathrm{D}$ therapy plannings were compared in terms of dosimetric parameters. The aim was to assess the field efficiency of the $2 \mathrm{D}$ plan and to evaluate the $3 \mathrm{D}$ plan in terms of both its coverage of the target volume and its effect to decrease the normal tissue doses.

\section{Materials and Methods}

This study is conducted using the information recorded in the treatment planning systems (TPS) for 36 patients with gastric adenocarcinoma who were treated with 3D conformal RT between February 2009 and July 2011 at Ankara University Medical School Radiation Oncology Department. All patients were underwent surgery. They were given adjuvant chemotherapy (every 28 days 5-6 cycles of $425 \mathrm{mg} / \mathrm{m}^{2} /$ day 5 -FU 5 days, folinic acid $20 \mathrm{mg}$ / $\mathrm{m}^{2}$ /day 5 days) and radiotherapy concurrently with the $2^{\text {nd }}$ cycle of the chemotherapy. A total of 45-50,4 Gy RT was applied, with a fraction dose of $1.8 \mathrm{~Gy} /$ day. Sixteen patients received 50.4 Gy and twenty patients received 45 Gy.

Clinical target volume (CTV) was defined according to the localization of the primary tumor, stage, proximity of the surgical border, number of involved and sampled lymph nodes and their localization. Preoperative CT images were used for the delineation of tumor bed and 
lymph nodes.

For PTV, a margin of $1 \mathrm{~cm}$ was added to planning computer in all directions. 3D planning was conducted on using the planning program of Precise Plan Release 2.16. We tried to maintain the dose discrepancy inside PTV between the $\pm 5 \%$ and $\pm 5 \%$ of the described dose. Fields were established in a manner that the dose of spinal cord would not exceed 46 Gy, liver V30 would not exceed $\% 70$ and finally kidney V20 would not exceed $30 \%$. All patients were underwent therapy on this plan using Linear accelerator (Elekta Synergy Platform) with photon energies of $6 \mathrm{MV}-18 \mathrm{MV}$

Conventional RT field was determined in a manner that it would cover gastric bed, perigastric, celiac, splenic, hepatic, pancreaticoduodenal and paraaortic lymph nodes and conventional field borders were placed over existing images on planning computer and two dimensional planning was carried out. For tumors localized in the proximal region and cardia, upper margin of the field was established to comprise 2/3 of the left hemidiaphragm, and the lower margin being below the L3 vertebra. For distally localized tumors the upper margin of the field was the dome of the diaphragm and lower margin was again below the L3 vertebra. Since the large part of the left kidney was covered inside the field, sparing of the right kidney was attempted as possible as. For the two dimensional planning,two fields were used being anterior-posterior and posterior-anterior (AP-PA). The same dose applied in 3D plan was used for each patient. Dose-volume histograms for each of the two plans were created.

DVHs of these two plans were compared for each patient in terms of the minimum, maximum and mean doses of PTV, right and left kidney, liver and spinal cord and the volume percentage of the PTV taken the scheduled dose, volume percentage of the each kidney taken 20 Gy (V20), volume percentage of the liver taken 30 Gy (V30) and volume percentage of the spinal cord received $>46 \mathrm{~Gy}$.

Normal tissue complication probabilities (NTCPs) were calculated for kidneys and liver. EUD (equivalant uniform dose) was used to calculate NTCP. For the transition from EUD to NTCP a formulation developed by Luxton et al. (2008) was used.

\section{Statistical Method}

Data analysis was performed using SPSS 11.5 for Windows. The distribution of continuous variables was assessed using Shapiro Wilk test. Significance of the difference for the measurements was assessed by paired t-test for normally distributed variables and by Wilcoxon signed rank test for variables which are not normally distributed. Results were admitted statistically significant for $\mathrm{p}<0.05$.

\section{Results}

For each of the 36 patients DVHs of both 2D and 3D plans were created. Using these DVHs, minimum, maximum and mean doses (as cGy), volume percentage of PTV receiving the scheduled dose, V20 of each kidney, V30 of liver and volume percentage of spinal cord receiving more than 46 Gy were calculated and compared for patients receiving 50.4 Gy (group A) and 45 Gy (group B) separately and for the whole patient group (group C) as well.

In group A ; minimum, maximum and mean doses of PTV, minimum and mean doses and V30 of liver were statistically higher on 3DRT; maximum doses of right and left kidney, minimum and mean doses of the left kidney, V20 of the left kidney, maximum and mean doses of spinal cord and its mean volume percentage receiving $46 \mathrm{~Gy}$ were observed as statistically higher on 2DRT (Table 1).

In group B, minimum, maximum and mean doses of PTV, minimum and maximum liver doses were statistically higher on 3DRT; maximum doses of right and left kidney, mean dose of left kidney and V20, maximum and mean doses of spinal cord and mean volume percentage receiving 46 Gy were statistically higher on 2DRT (Table 2).

In group $\mathrm{C}$, minimum, maximum and mean doses of PTV and mean percentage PTV volume receiving the scheduled dose, minimum and mean liver doses and its V30 were statistically higher on 3DRT; maximum doses kidneys, minimum, mean doses and V20 of left kidney; minimum, maximum and mean doses of spinal cord and is mean percentage spinal cord volume receiving $46 \mathrm{~Gy}$ were statistically higher on 2DRT. On 2DRT left kidney

\section{Table 1. Obtained Results Among Group A}

\begin{tabular}{|c|c|c|c|}
\hline Parameters & Plan & Results & p-value \\
\hline PTV & $2 \mathrm{D}$ & $286.50(88.00-5082.00)$ & $<0.001$ \\
\hline Minimum (cGy) & $3 \mathrm{D}$ & $3958.00(288.00-4705.00)$ & \\
\hline PTV & $2 \mathrm{D}$ & $5634.50(5335.00-5948.00)$ & 0.017 \\
\hline Maximum (cGy) & $3 \mathrm{D}$ & $5856.50(5506.00-6149.00)$ & \\
\hline PTV & $2 \mathrm{D}$ & $5022.00(4184.00-5420.00)$ & 0.003 \\
\hline Mean (cGy) & $3 \mathrm{D}$ & $5203.00(4869.00-5485.00)$ & \\
\hline PTV & $2 \mathrm{D}$ & $88.00(27.00-100.00)$ & 0.148 \\
\hline$\%$ Volume & $3 \mathrm{D}$ & $90.60(28.00-99.50)$ & \\
\hline Right kidney & $2 \mathrm{D}$ & $45.50(26.00-157.00)$ & 0.589 \\
\hline Minimum (cGy) & $3 \mathrm{D}$ & $51.50(0.00-183.00)$ & \\
\hline Right kidney & $2 \mathrm{D}$ & $5076.00(4440.00-5601.00)$ & 0.012 \\
\hline Maximum (cGy) & $3 \mathrm{D}$ & $4137.00(715.00-5732.00)$ & \\
\hline Right kidney & $2 \mathrm{D}$ & $576.00(157.00-1064.00)$ & 0.955 \\
\hline Mean (cGy) & $3 \mathrm{D}$ & $494.00(68.00-1959.00)$ & \\
\hline Right kidney & $2 \mathrm{D}$ & $11.80(3.50-32.00)$ & 0.535 \\
\hline$\%$ volume & $3 \mathrm{D}$ & $7.55(0.00-37.70)$ & \\
\hline Left kidney & $2 \mathrm{D}$ & $158.00(116.00-201.00)$ & 0.027 \\
\hline Minimum (cGy) & $3 \mathrm{D}$ & $81.50(0.00-815.00)$ & \\
\hline Left kidney & $2 \mathrm{D}$ & $5380.50(5269.0-5576.0)$ & 0.006 \\
\hline Maximum (cGy) & $3 \mathrm{D}$ & $5157.00(717.00-5675.00)$ & \\
\hline Left kidney & $2 \mathrm{D}$ & $2657.87 \pm 583.51$ & $<0.001$ \\
\hline Mean (cGy) & $3 \mathrm{D}$ & $1121.56 \pm 715.69$ & \\
\hline Left kidney & $2 \mathrm{D}$ & $50.15(28.40-75.70)$ & $<0.001$ \\
\hline$\%$ volume & $3 \mathrm{D}$ & $16.00(0.00-43.20)$ & \\
\hline Spinal cord & $2 \mathrm{D}$ & $77.50(0.00-282.00)$ & 0.064 \\
\hline Minimum (cGy) & $3 \mathrm{D}$ & $12.00(0.00-906.00)$ & \\
\hline Spinal cord & $2 \mathrm{D}$ & $5522.50(5271.00-5731.00)$ & $<0.001$ \\
\hline Maximum (cGy) & $3 \mathrm{D}$ & $4226.00(1412.00-5569.00)$ & \\
\hline Spinal cord & $2 \mathrm{D}$ & $3753.44 \pm 587.75$ & $<0.001$ \\
\hline Mean (cGy) & $3 \mathrm{D}$ & $1746.37 \pm 954.74$ & \\
\hline Spinal cord & $2 \mathrm{D}$ & $64.75(40.00-82.30)$ & $<0.001$ \\
\hline$\%$ volume & $3 \mathrm{D}$ & $0.00(0.00-0.00)$ & \\
\hline Liver & $2 \mathrm{D}$ & $23.50(0.00-64.00)$ & 0.003 \\
\hline Minimum (cGy) & $3 \mathrm{D}$ & $64.50(0.00-165.00)$ & \\
\hline Liver & $2 \mathrm{D}$ & $5422.00(5079.00-5662.00)$ & 0.427 \\
\hline Maximum (cGy) & $3 \mathrm{D}$ & $5421.50(5097.00-6003.00)$ & \\
\hline Liver & $2 \mathrm{D}$ & $1344.06 \pm 270.45$ & $<0.001$ \\
\hline Mean (cGy) & $3 \mathrm{D}$ & $1992.00 \pm 559.39$ & \\
\hline Liver & $2 \mathrm{D}$ & $21.31 \pm 4.71$ & 0.034 \\
\hline$\%$ volume & $3 \mathrm{D}$ & $31.59 \pm 18.30$ & \\
\hline
\end{tabular}


Table 2. Obtained Results Among Group B

\begin{tabular}{|c|c|c|c|}
\hline Parameters & Plan & Results & p-value \\
\hline PTV & $2 \mathrm{D}$ & $151.00(0.00-4242.00)$ & $<0.001$ \\
\hline Minimum (cGy) & $3 \mathrm{D}$ & $3937.50(0.00-4344.00)$ & \\
\hline PTV & $2 \mathrm{D}$ & $5120.50(4742.00-5513.00)$ & 0.255 \\
\hline Maximum (cGy) & $3 \mathrm{D}$ & $5133.00(4932.00-5745.00)$ & \\
\hline PTV & $2 \mathrm{D}$ & $4420.50(3196.00-4660.00)$ & $<0.001$ \\
\hline Mean (cGy) & $3 \mathrm{D}$ & $4704.50(4583.00-4906.00)$ & \\
\hline PTV & $2 \mathrm{D}$ & $78.90(42.00-92.70)$ & $<0.001$ \\
\hline$\%$ volume & $3 \mathrm{D}$ & $93.30(80.00-99.10)$ & \\
\hline Right kidney & $2 \mathrm{D}$ & $44.50(23.00-140.00)$ & 0.391 \\
\hline Minimum (cGy) & $3 \mathrm{D}$ & $61.00(0.00-245.00)$ & \\
\hline Right kidney & $2 \mathrm{D}$ & $4603.00(4338.00-5008.00)$ & 0.015 \\
\hline Maximum (cGy) & $3 \mathrm{D}$ & $4282.50(72.00-5118.00)$ & \\
\hline Right kidney & $2 \mathrm{D}$ & $633.00(303.00-1521.00)$ & 0.502 \\
\hline Mean (cGy) & $3 \mathrm{D}$ & $873.50(25.00-2068.00)$ & \\
\hline Right kidney & $2 \mathrm{D}$ & $9.15(3.20-31.50)$ & 0.341 \\
\hline \%volume & $3 \mathrm{D}$ & $15.75(0.00-52.50)$ & \\
\hline Left kidney & $2 \mathrm{D}$ & $148.50(50.00-207.00)$ & 0.052 \\
\hline Minimum (cGy) & $3 \mathrm{D}$ & $76.50(0.00-1074.00)$ & \\
\hline Left kidney & $2 \mathrm{D}$ & $4844.00(4631.00-5214.00)$ & $<0.001$ \\
\hline Maximum (cGy) & 3D & $4611.50(3469.00-4901.00)$ & \\
\hline Left kidney & $2 \mathrm{D}$ & $2471.20 \pm 757.01$ & $<0.001$ \\
\hline Mean (cGy) & $3 \mathrm{D}$ & $1464.95 \pm 785.28$ & \\
\hline Left kidney & $2 \mathrm{D}$ & $48.35(20.30-84.50)$ & 0.005 \\
\hline$\%$ volume & $3 \mathrm{D}$ & $26.00(0.6071 .00)$ & \\
\hline Spinal cord & $2 \mathrm{D}$ & $134.50(0.00-4443.00)$ & 0.064 \\
\hline Minimum (cGy) & 3D & $54.50(0.00-1109.00)$ & \\
\hline Spinal cord & $2 \mathrm{D}$ & $4888.00(4692.00-5546.00)$ & $<0.001$ \\
\hline Maximum (cGy) & $3 \mathrm{D}$ & $4194.50(1255.00-4825.00)$ & \\
\hline Spinal cord & $2 \mathrm{D}$ & $3548.20 \pm 675.68$ & $<0.001$ \\
\hline Mean (cGy) & 3D & $1897.00 \pm 967.87$ & \\
\hline Spinal cord & $2 \mathrm{D}$ & $62.95(7.00-90.00)$ & $<0.001$ \\
\hline$\%$ volume & $3 \mathrm{D}$ & $0.00(0.00-16.00)$ & \\
\hline Liver & $2 \mathrm{D}$ & $20.50(0.00-25.00)$ & $<0.001$ \\
\hline Minimum (cGy) & $3 \mathrm{D}$ & $60.50(16.00-5403.00)$ & \\
\hline Liver & $2 \mathrm{D}$ & $4759.50(4417.00-5143.00)$ & 0.167 \\
\hline Maximum (cGy) & 3D & $4974.50(2852.00-5313.00)$ & \\
\hline Liver & $2 \mathrm{D}$ & $1204.55 \pm 387.88$ & $<0.001$ \\
\hline Mean (cGy) & $3 \mathrm{D}$ & $2023.80 \pm 479.24$ & \\
\hline Liver & $2 \mathrm{D}$ & $21.66 \pm 8.39$ & 0.051 \\
\hline$\%$ volume & $3 \mathrm{D}$ & $27.88 \pm 12.03$ & \\
\hline
\end{tabular}

volume covered in the treatment site is quite large then sparing of the right kidney is envisaged as possible as, the mean doses of the right kidney are lower on $2 \mathrm{D}$, but not statistically significant. Liver doses and volumes appear to be lower on 2DRT because lymphatic sites are limitedly considered on right side and the liver volume covered in the treatment site is small in AP fields. However on 3D planning, lymphatic regions that are drawn detailed according to tumor localization caused an enlargement on right side and liver volume in treatment site (Table 3 ).

Table 1, 2 and 3 shows obtained results on $2 \mathrm{D}$ and 3D plannings.

For the NTCP calculations made for kidneys and liver, NTCP appeared to be significantly high for liver on 3D and for left kidney on 2D whereas no statistically significant difference was observed for right kidney. NTCP results are shown in Table 4.

\section{Discussion}

Postoperative chemoradiotherapy is established as the standard treatment in stage IB-IV, M0 gastric cancer. Tumor bed, anastomosis line and regional lymph nodes should be covered in the RT field since they are risk-bearing structures for recurrence. Because of the surrounding
Table 3. Obtained Results Among Group C

\begin{tabular}{|c|c|c|c|}
\hline Parameters & Plan & Results (cGy) & p-value \\
\hline PTV & $2 \mathrm{D}$ & $183.00(0.00-5082.00)$ & $<0.001$ \\
\hline Minimum (cGy) & $3 \mathrm{D}$ & $3937.50(0.00-4705.00)$ & \\
\hline PTV & $2 \mathrm{D}$ & $5336.50(4742.00-5948.00)$ & 0.009 \\
\hline Maximum (cGy) & $3 \mathrm{D}$ & $5428.50(4932.00-6149.00)$ & \\
\hline PTV & $2 \mathrm{D}$ & $4503.50(3196.00-5420.00)$ & $<0.001$ \\
\hline Mean (cGy) & $3 \mathrm{D}$ & $4875.00(4583.00-5485.00)$ & \\
\hline PTV & $2 \mathrm{D}$ & $80.00(27.00-100.00)$ & $<0.001$ \\
\hline$\%$ volume & $3 \mathrm{D}$ & $92.70(28.00-99.50)$ & \\
\hline Right kidney & $2 \mathrm{D}$ & $44.50(23.00-157.00)$ & 0.283 \\
\hline Minimum (cGy) & $3 \mathrm{D}$ & $59.00(0.00-245.00)$ & \\
\hline Right kidney & $2 \mathrm{D}$ & $4739.50(4338.00-5601.00)$ & $<0.001$ \\
\hline Maximum (cGy) & $3 \mathrm{D}$ & $4282.50(72.00-5732.00)$ & \\
\hline Right kidney & $2 \mathrm{D}$ & $617.00(157.00-1521.00)$ & 0.578 \\
\hline Mean (cGy) & $3 \mathrm{D}$ & $660.00(25.00-2068.00)$ & \\
\hline Right kidney & $2 \mathrm{D}$ & $10.40(3.20-32.00)$ & 0.712 \\
\hline$\%$ volume & $3 \mathrm{D}$ & $9.35(0.00-52.50)$ & \\
\hline Left kidney & $2 \mathrm{D}$ & $151.00(50.00-207.00)$ & 0.005 \\
\hline Minimum (cGy) & $3 \mathrm{D}$ & $79.50(0.00-1074.00)$ & \\
\hline Left kidney & $2 \mathrm{D}$ & $5064.00(4631.00-5576.00)$ & $<0.001$ \\
\hline Maximum (cGy) & $3 \mathrm{D}$ & $4664.50(717.00-5675.00)$ & \\
\hline Left kidney & $2 \mathrm{D}$ & $2554.17 \pm 682.55$ & $<0.001$ \\
\hline Mean (cGy) & $3 \mathrm{D}$ & $1312.33 \pm 764.35$ & \\
\hline Left kidney & $2 \mathrm{D}$ & $48.80(20.30-84.50)$ & $<0.001$ \\
\hline$\%$ volume & $3 \mathrm{D}$ & $22.05(0.00-71.00)$ & \\
\hline Spinal cord & $2 \mathrm{D}$ & $109.00(0.00-4443.00)$ & 0.010 \\
\hline Minimum (cGy) & $3 \mathrm{D}$ & $39.00(0.00-1109.00)$ & \\
\hline Spinal cord & $2 \mathrm{D}$ & $5186.50(4692.00-5731.00)$ & $<0.001$ \\
\hline Maximum (cGy) & $3 \mathrm{D}$ & $4199.50(1255.00-5569.00)$ & \\
\hline Spinal cord & $2 \mathrm{D}$ & $3639.41 \pm 637.64$ & $<0.001$ \\
\hline Mean (cGy) & $3 \mathrm{D}$ & $1830.06 \pm 951.29$ & \\
\hline Spinal cord & $2 \mathrm{D}$ & $64.75(7.00-90.00)$ & $<0.001$ \\
\hline$\%$ volume & $3 \mathrm{D}$ & $0.00(0.00-16.00)$ & \\
\hline Liver & $2 \mathrm{D}$ & $21.50(0.00-64.00)$ & $<0.001$ \\
\hline Minimum (cGy) & $3 \mathrm{D}$ & $61.00(0.00-5403.00)$ & \\
\hline Liver & $2 \mathrm{D}$ & $5047.0(4417.0-5662.0)$ & 0.055 \\
\hline Maximum (cGy) & $3 \mathrm{D}$ & $5132.00(2852.00-6003.00)$ & \\
\hline Liver & $2 \mathrm{D}$ & $1266.56 \pm 343.46$ & $<0.001$ \\
\hline Mean (cGy) & $3 \mathrm{D}$ & $2009.67 \pm 508.96$ & \\
\hline Liver & $2 \mathrm{D}$ & $21.51 \pm 6.91$ & 0.003 \\
\hline$\%$ volume & $3 \mathrm{D}$ & $29.53 \pm 15.02$ & \\
\hline
\end{tabular}

Table 4. NTCP Results

\begin{tabular}{llcr}
\hline Parameters & Plan & Results & p-value \\
\hline Liver & 2D & $0.0062(0.0024-0.0083)$ & $<0.001$ \\
& 3D & $0.0086(0.0052-0.1064)$ & \\
Right kidney & 2D & $0.0032(0.0010-0.0073)$ & 0.682 \\
& 3D & $0.0033(0.0001-0.0098)$ & \\
Left kidney & 2D & $0.1335(0.0051-1.9500)$ & $<0.001$ \\
& 3D & $0.0054(0.0005-0.9692)$ & \\
\hline
\end{tabular}

critical organs with low tolerance, radiotherapy is a critical process in gastric cancer (Henning et al., 2000; Baeza et al., 2001; Macdonald et al., 2005; Hazard et al. 2006; Pemberton et al., 2006).

This study was conducted to compare two distinct planning methods for gastric cancer radiotherapy planning in terms of organ at risk doses and PTV doses. Three dimensional and two dimensional therapy planning were compared and a more favorable dose distribution for PTV and statistically lower doses for left kidney and spinal cord and a lower maximum dose for right kidney were observed on $3 \mathrm{D}$ conformal plan when compared with 2D AP-PA technique whereas liver doses were statistically higher.

Baeza et al. (2001) preferred to use AP-PA field for the radiotherapy in gastric cancer patients and suggested that this treatment modality was safe and easy because 
of the possibility of sparing kidneys. For the treatments focusing only on the organ at risk without considering target volumes, target volume may not receive the anticipated therapy, yet in our study we observed a better dose distribution for PTV using 3D compared to AP-PA therapy plan. Besides its mean doses, minimum doses of PTV appeared to be higher on 3D plan. Mean minimum dose of PTV is below 1000 cGy on 2D plan, in other words, there are some sites receiving $<1000 \mathrm{cGy}$ inside PTV, thus a decrease in tumor control and in expected local control rate may occur.

Henning et al. (2000) retrospectively assessed 63 patients who had received chemoradiotherapy postoperatively and found a higher toxicity rate in patients treated using AP-PA technique compared with patients given multi-field therapy. Caudry et al. (1987) compared AP-PA technique to the three-field technique and reported a lower dose for left kidney in three-field technique but a higher dose for liver. In this study the AP two-field technique was used in 2D planning and lower liver doses were obtained in this plan using two fields. This was an expected situation because liver receives less radiation in the AP fields but in multi-field plans used to obtain a better dose distribution, liver receives higher doses because of the fields created at the right side. In our study though the liver doses were observed higher on 3D, they were still within the tolerance dose range.

Marcenaro et al. (2006) compared AP-PA technique to the multi-field technique and they found a lower dose for left kidney and higher dose for liver, as observed in our study. Though the liver dose was higher in the multifield, it did not exceed the tolerance dose range, as seen in our study.

In a study conducted by El-Hossiny et al. (2009) 2D (AP-PA field) and 3D plans were compared in 17 patients with stage II-III gastric cancer and a lower dose for left kidney and spinal cord was observed on 3D; but the liver dose was higher. In another study Soyfer et al. (2007) compared 2D (AP-PA field) and 3D (four-field technique) in 19 patients and observed a lower dose for kidney and spinal cord on 3D while the liver dose was higher. A study by Leong et al. (2005) comparing 2D (AP-PA field) and $3 \mathrm{D}$ plans in 15 patients with stage II-IV gastric cancer revealed a lower kidney and spinal cord dose on 3D. In this study kidney volumes receiving $20 \mathrm{~Gy}$ were higher on $2 \mathrm{D}$ while liver volumes receiving 30 were higher on 3D. In our study, right kidney and spinal cord doses and maximum kidney doses were also lower on 3D planning. No statistically significant difference was found between minimum and mean doses of right kidney. Left kidney volume receiving $20 \mathrm{~Gy}$ was lower on $3 \mathrm{D}$ while no statistically significant difference was found between the two plans in terms of right kidney volume receiving 20 Gy; liver volume receiving $30 \mathrm{~Gy}$ was higher on $3 \mathrm{D}$. The reason that there were no difference between the minimum and mean doses of right kidney may be the sparing of the right kidney since the large part of the left one was covered in the RT field. Moreover left kidney doses and left kidney volume receiving $20 \mathrm{~Gy}$ was above the tolerance limits on $2 \mathrm{D}$ in all patient groups. The mean kidney dose that should be limited at 18 Gy was above this value in both $4500 \mathrm{cGy}$ and 5040 cGy groups. While the kidney volume receiving 20 Gy should be lower than $30 \%$, this value appeared in both 5040 cGy and 4500 cGy groups higher than $50 \%$ on 2D. This could be accepted as a factor increasing the risk of toxicity in patients. Relatively high spinal cord doses on anterior-posterior fields are anticipated and since spinal cord can be spared with ease on multiple-field, spinal cord doses were found to be lower on 3D planning. Though the liver doses and liver volume receiving $30 \mathrm{~Gy}$ were higher on 3D, they were still within the tolerance limits.

Wals et al. (2006) assessed NTCP and TCP in 29 patients underwent post-operative chemoradiotherapy. Fourteen of these 20 patients underwent 2D and the remaining 15 patients underwent $3 \mathrm{D}$ planning and these two patient groups were compared for TCP and NTCP. Percentage NTCPs were higher on 2D for left kidney, liver and spinal cord. In our study NTCP was higher for left kidney on 2D, and higher for liver on $3 \mathrm{D}$, thus, it is consistent with the finding of higher left kidney dose on 2D and higher liver dose on 3D planning.

Technological advances brought IMRT (intensity modulated radiation therapy) into the agenda for gastric cancer radiotherapy. In a study by Lohr et al. (2003) comparing 3D planning and IMRT, liver and kidney doses were lower in IMRT. But Chang et al. (2008) did not reveal any dosimetric advantage of IMRT over 3D planning. In a study conducted by Minn et al. (2010) liver doses were lower with IMRT while no statistically significant difference was observed for liver and PTV doses. Milano et al. (2006) compared 2D, 3D and IMRT; PTV dose appeared better with IMRT and kidney and liver doses were lower. In a study by Alani et al. (2009) comparing 3D and IMRT no significant difference was observed for PTV doses, IMRT was only marginally better than 3D conformal radiotherapy at protecting spinal cord and kidneys from radiation. Though there is no explicit advantage of IMRT in PTV dose distribution when compared to 3D planning, because its normal tissue doses appeared to be lower, IMRT may be preferred for gastric cancer RT in experienced centers.

In conclusion, it can be suggested that 3D therapy planning would provide a better tumor control and would result in fewer complications over 2D therapy because of low maximum right kidney dose and low left kidney and spinal cord doses and liver doses being within tolerance dose despite high levels seen on 3D and a significantly good PTV volume and PTV mean doses. Long-term follow-up of patients and clinical demonstration of these results are critical for the success of irradiations using advanced technology.

\section{References}

Alani S, Soyfer V, Strauss N, et al (2009). Limited advantages of IMRT over 3D conformal radiation therapy in the management of gastric cancer. Int J Radiat Biol Phys, 74, $562-6$

Baeza MR, Giannini TO, Riviera SR, et al (2001). Adjuvan radiochemotherapy in the treatment of completely resected, locally advanced gastric cancer. Int J Radiat Oncol Biol Phys, 50, 645-50

Calik M, Calik I, Demirci E, et al (2014). Goseki grade and 
tumour location influnce survival of patients with gastric cancer. Asian Pac J Cancer Prev, 15, 1429-34.

Caudry M, Escarmant P, Maire JP, et al (1987). Radiotherapy of gastric cancer with three field combination: feasibility, tolerance and survival. Int J Radiat Oncol Biol Phys, 13, 1821-7.

Chung HT, Lee B, Park E, et al (2008). Can all centers plan IMRT effectively? an external audit of dosimetric comparisons between three-dimensional conformal radiotherapy and imrt for adjuvan chemoradiation for gastric cancer. Int $J$ Radiat Biol Phys, 71, 1167-74.

Czito BG, Palta M, Willett CG (2013). Stomach Cancer. In:Halperin EC, Perez CA, Brady LW. Perez and Brady's principles and practice of radiation oncology. $6^{\text {th }}$ ed.philadelphia: lippincott williams and wilkins, 1165-89.

El-Hossiny HA, Diab NA, El-Taher MM (2009). A comparative dosimetric study of adjuvant 3D conformal radiotherapy for operable stomach cancer versus AP-PA conventional radiotherapy in NCI-Cairo. J Egypt Natl Canc Inst, 21, 197-202.

Hazard L, O'connor J, Scaife C (2006). Role of radiation therapy in gastric carcinoma. World J Gastroenterol, 12, 1511-20.

Henning GT, Schild SE, Stafford SL, et al (2000). Results of irradiation or chemoirradiation following resection of gastric adenocarcinoma. Int J Radiat Oncol Biol Phys, 46, 589-98.

Leong T, Willis D, Joon DL, et al (2005). 3D Conformal radiotherapy for gastric cancer-result of a comparative planning study. Radiotherapy Oncolog, 74, 301-6.

Lohr F, Dobler B, Mai S, et al (2003) Optimization of dose distributions for adjuvan locoregional radiotherapy of gastric cancer by IMRT. Strahlenther Oncologie, 179, 557-63.

Luxton G, Keall PJ, King CR (2005). A new formula for normal tissue complication probability (NTCP) as a function of equivalent uniform dose (EUD) phys. Med Biol, 53, 23-36.

Macdonald SJ (2005). Role of post-operative chemoradiation in resected gastric cancer. J Surg Oncol, 90, 166-70.

Marcenora M, Foppiano F, Durzu S, et al (2006). Kidney-sparing radiotherapy by multiple-field three-dimensional technique in the postoperative management of patients with gastric cancer: comparison with standart two-field conformal technique. Tumori, 92, 34-40.

Milano MT, Garofalo MC, Chmura J, et al (2006). IMRT in the treatment of gastric cancer: early clinical outcome and dosimetric comparison with conventional techniques. $\mathrm{Br} \mathrm{J}$ Radiol, 79, 497-50.

Minn Y, Hsu A, La T, et al (2010).Comparison of IMRT and 3- dimensional conformal radiotherapy for gastric cancer. Cancer, 116, 3943-52.

Oh MG, Kim JH, Han MA, et al (2014). Family history and survival of patients with gastric cancer: a meta-analysis. Asian Pac J Cancer Prev, 15, 3465-70

Pemberton L, Coote J, Perry L, et al (2006). Adjuvant chemoradiotherapy for gastric carcinoma: dosimetric i mplications of conventional gastric bed irradiation and toxicity. Clinical Oncology, 18, 663-8.

Smalley SR, Benedetti JK, Haller DG, et al (2012). Updated analysis of SWOG-directed intergroup study 0116: a phase III trial of adjuvant radiochemotherapy versus observation after curative gastric cancer resection. J Clinical Oncol, 30, 2327-33

Soyfer V, Corn BW, Melamud A, et al (2007). Three-dimensional non-coplanar conformal radiotherapy yields better results than traditional beam arrangements for adjuvant treatment of gastric cancer. Int J Radiat Oncol Biol Phys, 69, 364-9.

Wals A, Contreras J, Macias J, et al (2006). Damage assesment in gastric cancer treatment with adjuvan radiochemotherapy: calculation of the NTCP's from differential HDV of the
Organs at Risk. Clin Trans Oncol, 8, 271-8.

Zare A, Mahmoodi M, Mohammad K, et al (2013). Survival analysis of patients with gastric cancer undergoing surgery at Iran cancer institute: a method based on multi-state models. Asian Pac J Cancer Prev, 14, 6369-73. 